\title{
Measurements of the dimensions of the aortic and pulmonary pathways in the human fetus: a correlative echocardiographic and morphometric study
}

\author{
ANNALISA ANGELINI, * LINDSEY D ALLAN, $†$ ROBERT H ANDERSON, * \\ DIANE C CRAWFORD, $\dagger$ SUNDER K CHITA, $\dagger$ SIEW YEN HO* \\ From the ${ }^{\star}$ Department of Paediatrics, Cardiothoracic Institute, Brompton Hospital; and the $\dagger$ British Heart \\ Foundation Research Centre for Perinatal Cardiology, Guy's Hospital, London
}

SUMMARY Thirty normal hearts from fetuses aborted at 10-33 weeks' gestation were measured directly and the results were compared with echocardiographic measurements in 20 normal live fetuses of 23-27 weeks' gestation. The diameters of the aorta, aortic isthmus, pulmonary arteries, and arterial duct were measured at standard levels and expressed as a ratio of the diameter of the ascending aorta. The ratios for the isthmus, the duct, and the right pulmonary artery were: 0.63 $(0.11), 0.51(0.13), 0.56(0.10)$ respectively for the direct measurement and $0.73(0.07), 0.7(0.08)$, $0.62(0.06)$ respectively at echocardiography. These results show that in mid-gestation the isthmus of the human fetus is not as small as that reported in animal models. This indicates that the distribution of the fetal circulation may not be the same either.

Our present knowledge of fetal circulation is derived mainly from studies carried out on animal models, particularly in lambs. Few studies have been performed in human fetuses. ${ }^{1-3}$ The experimental procedures used to study the lamb may have affected the physiological patterns. Studies were first performed on exteriorised fetal animals ${ }^{47}$ and, subsequently, in utero. ${ }^{89}$ The results of these studies led to the "flow theory", which directly relates the capacity of blood vessels to their relative sizes. ${ }^{10}$ This relation was subsequently extended as the "flowdependence rule"11 which hypothesised that, if the capacity of blood vessels is directly related to their cross sectional area, the sum of the squared diameters of the arterial branches should be equal to the square of the diameter of the arterial stem itself.

These haemodynamic indices of the fetal circulation derived from studies in animals have subsequently been extensively extrapolated to the human fetus, with adjustment only for the greater proportion of blood received by the human brain. In

Requests for reprints to Professor Robert H Anderson, Department of Paediatrics, Cardiothoracic Institute, Fulham Road, London SW3 6HP.

Accepted for publication 5 May 1988 this way, it has been calculated that the diameter of the human aortic isthmus is only about seven tenths that of the remainder of the aorta. It has further been presumed that the isthmal segment of the aortic arch is considerably narrower than the arterial duct (ductus arteriosus) and that the pulmonary arteries themselves are narrower still. Recent echocardiographic observations during mid-gestation on developing and normal human fetuses in our laboratories, however, suggest that the dimensions of

Table Age distribution of the fetuses studied

\begin{tabular}{lll}
\hline Weeks of gestation & Necropsy & Echocardiography \\
\hline 10 & 2 & \\
14 & 3 & \\
15 & 1 & \\
16 & 2 & \\
17 & 3 & \\
18 & 3 & \\
20 & 6 & 2 \\
21 & 1 & 9 \\
23 & 5 & 6 \\
24 & & 2 \\
25 & 1 & 1 \\
26 & 1 & \\
27 & 2 & \\
33 & & \\
Unknown & & \\
\hline
\end{tabular}




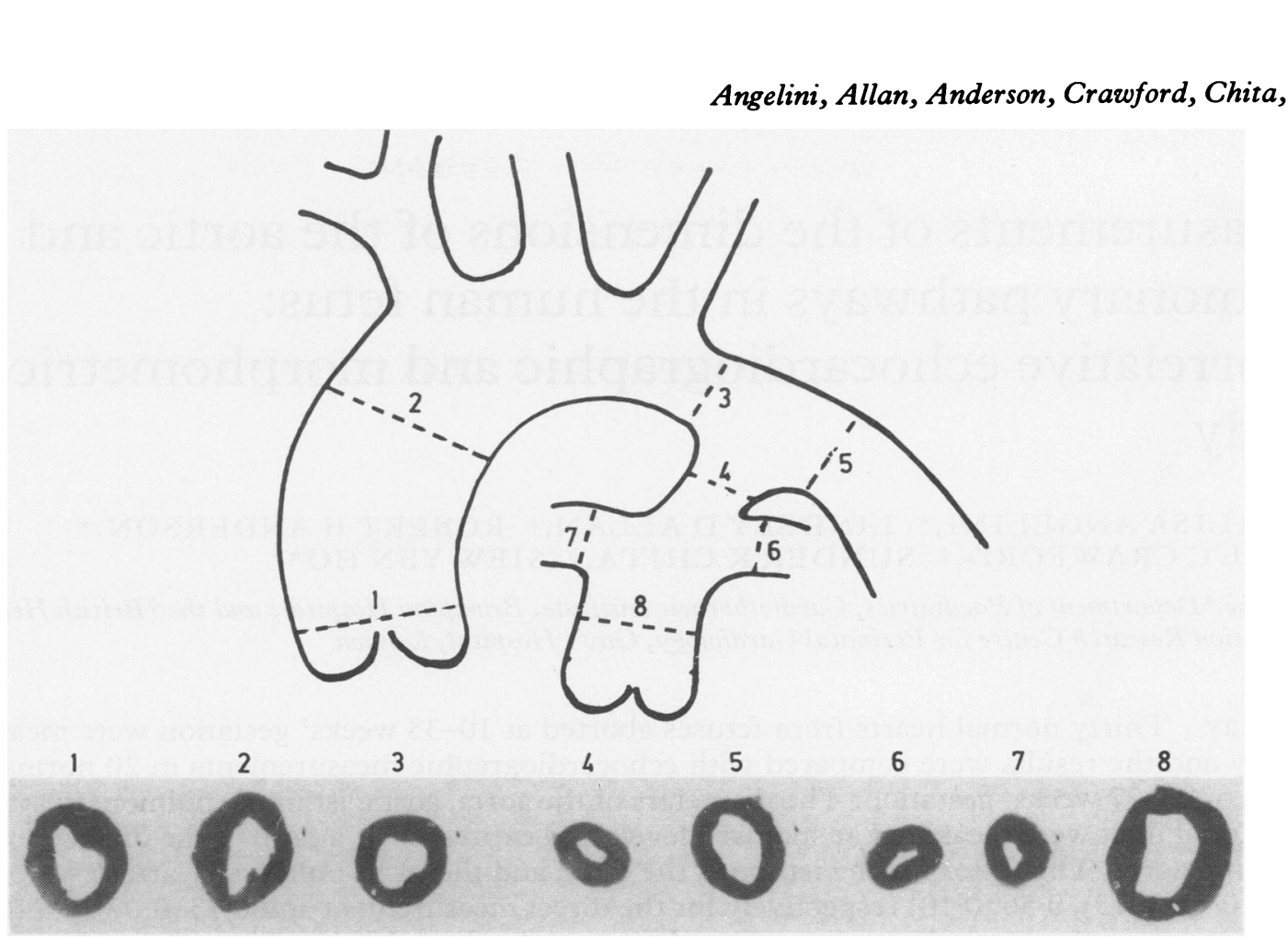

Fig 1 Levels of the arterial pathways at which the luminal diameters were assessed. The corresponding slices are shown below. (1) ascending aorta, (2) ascending aorta before the origin of the brachiocephalic artery, (3) isthmus, (4) arterial duct, (5) descending aorta, (6) left pulmonary artery, (7) right pulmonary artery, (8) pulmonary trunk.

the isthmus and pulmonary arteries are considerably greater than would be assumed according to the flow hypothesis that is based on measurements taken in fetal lambs. To investigate further this potential discrepancy, we performed echocardiographic and postmortem morphometric studies of the diameters of the aortic and pulmonary arterial pathways in two groups of normal human fetuses.

\section{Patients and methods}

\section{ABORTED FETUSES}

We studied the hearts and great vessels of 30 normal human fetuses after cardiovascular abnormalities were excluded at necropsy. The gestational age ranged from 10 to 33 weeks; in most it was 10 to 23 weeks (table). Because the drugs used to induce abortions may affect the wall of the arterial duct we studied only fetuses that had aborted spontaneously. The great vessels were separated from the heart and lungs. Twenty seven specimens were assessed after fixation in a $4 \%$ formaldehyde solution for at least 24 hours. The other three specimens were assessed both before and after fixation. We cut $300 \mu \mathrm{m}$ thick slices of each specimen at standard levels of the arterial pathways (fig 1). The slices were examined under light microscope connected to a BBC computer and 9 graphics table. We used this apparatus to measure theperimeters of the lumens. The luminal diameters

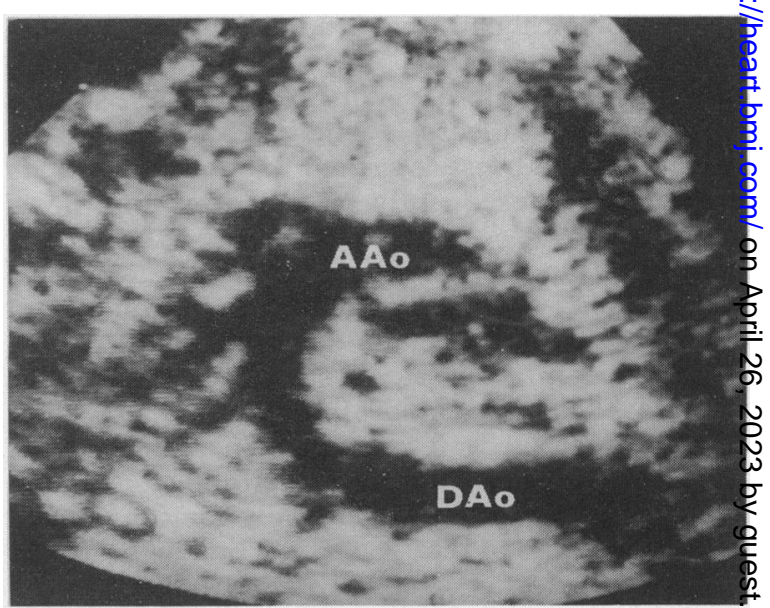

Fig 2 Echocardiogram (long axis view of the aortic arch) showing the uniform dimension of the aortic arch with only minimal narrowing at the site of the isthmus. AAo, ascending aorta; DAo, descending aorta. 


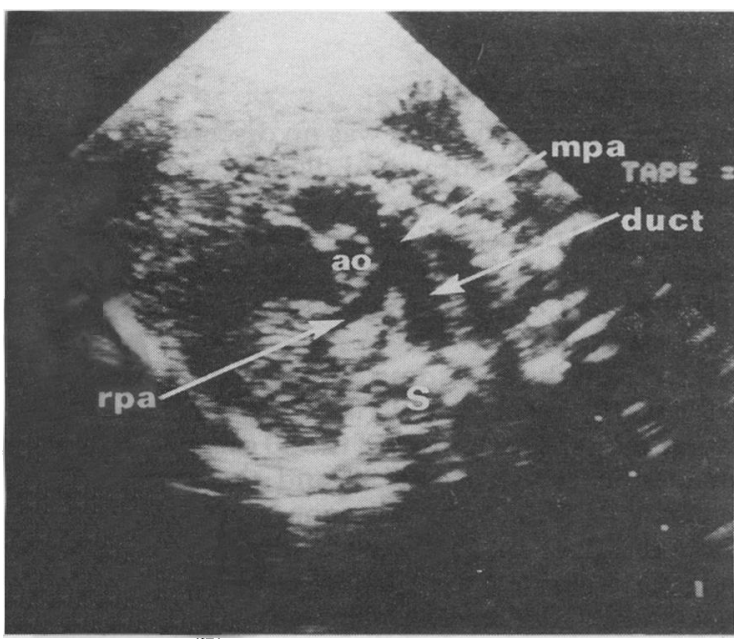

Fig 3 Echocardiogram (short axis view of the great arteries) showing the size of the arterial duct (duct) and the pulmomary trunk (mpa). ao, aorta; rpa, right pulmonary artery; s, spine.

were then derived by assuming that the vessels were circular in cross section. We took the mean of two measurements. Because the size of the specimens varied, we calculated the ratio between the diameter of each vessel and that of the ascending aorta. We chose the ascending aorta as the "control" so that we could compare postmortem morphometric data with the echocardiographic data (see below). We also expressed the diameter of the vessels in terms of the

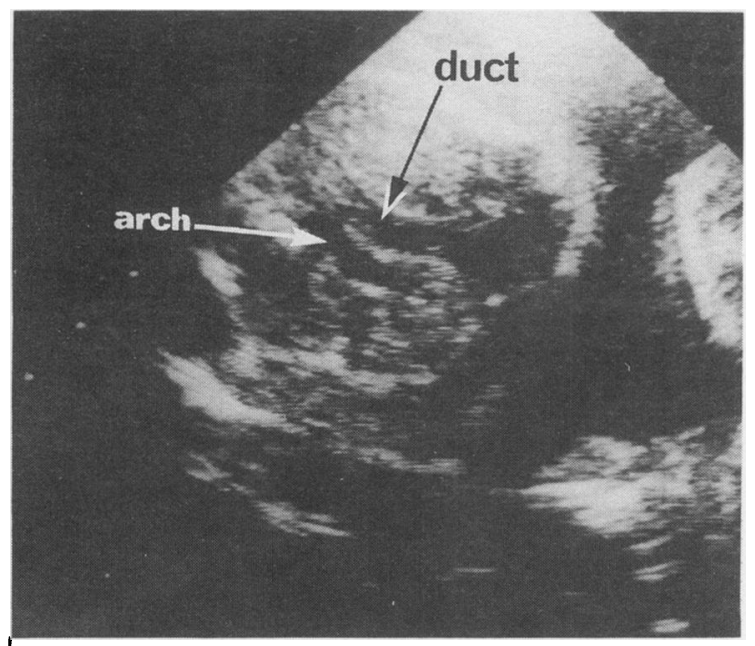

Fig 4 Echocardiogram (oblique horizontal view of the upper thorax showing the arch and duct in same section) taken obliquely across the thoracic inlet showing how, whenever possible, the arterial duct and the isthmus of the aortic arch were viewed in the same place.

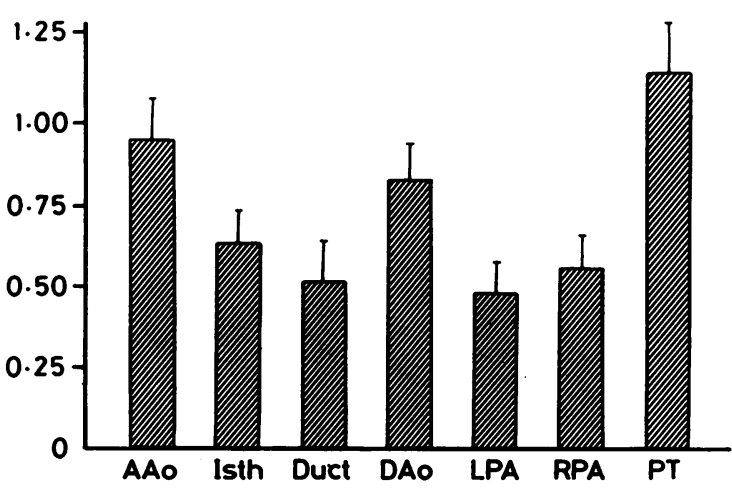

Fig 5 Histogram showing the mean (SD) of the ratio between the diameter of each vessel and that of the ascending aorta as determined from the postmortem data. AAo, ascending aorta before the origin of the brachiocephalic arteries; Isth, isthmus; duct, arterial duct; DAo, descending aorta; $L P A$, left pulmonary artery; $R P A$, right pulmonary artery; $P T$, pulmonary trunk.

descending aorta. We checked the validity of the flow-dependence rule, ${ }^{11}$ which states that the sum of the squared diameters of the arterial branches is equal to the square of the diameter of the arterial stem itself. We expressed the results as squared diameters and calculated their linear regression to establish the relation between the isthmus, the arterial duct, and the descending aorta and also that of the pulmonary trunk and its branches.

\section{ECHOCARDIOGRAPHIC STUDY}

We studied the fetuses of 20 women attending our department for diagnostic fetal echocardiography who were studied between 23 and 27 weeks' gestation. They were selected on the basis of the image quality of the echocardiographic study. All fetuses were healthy at delivery and subsequently.

We used the Hewlett Packard 77020 AC and a 5 $\mathrm{MHz}$ medium focused probe for echocardiography. This system has an axial resolution of $0.9 \mathrm{~mm}$ and a lateral resolution of $1.4 \mathrm{~mm}$. All patients had good quality images with the regions of the arch, duct, and isthmus being clearly visualised in several projections (figs 2 and 3 ). Only the dimensions of the descending aorta changed during the cardiac cycle. In the remainder of the arch and in the duct and pulmonary arteries the calibre of the vessels was more or less constant during systole and diastole. The ascending aorta was similarly of constant diameter and it, therefore, was chosen for purposes of comparison. All fetuses had a structurally normal heart as proven during fetal echocardiography and at postnatal follow up. The table shows the distribution 


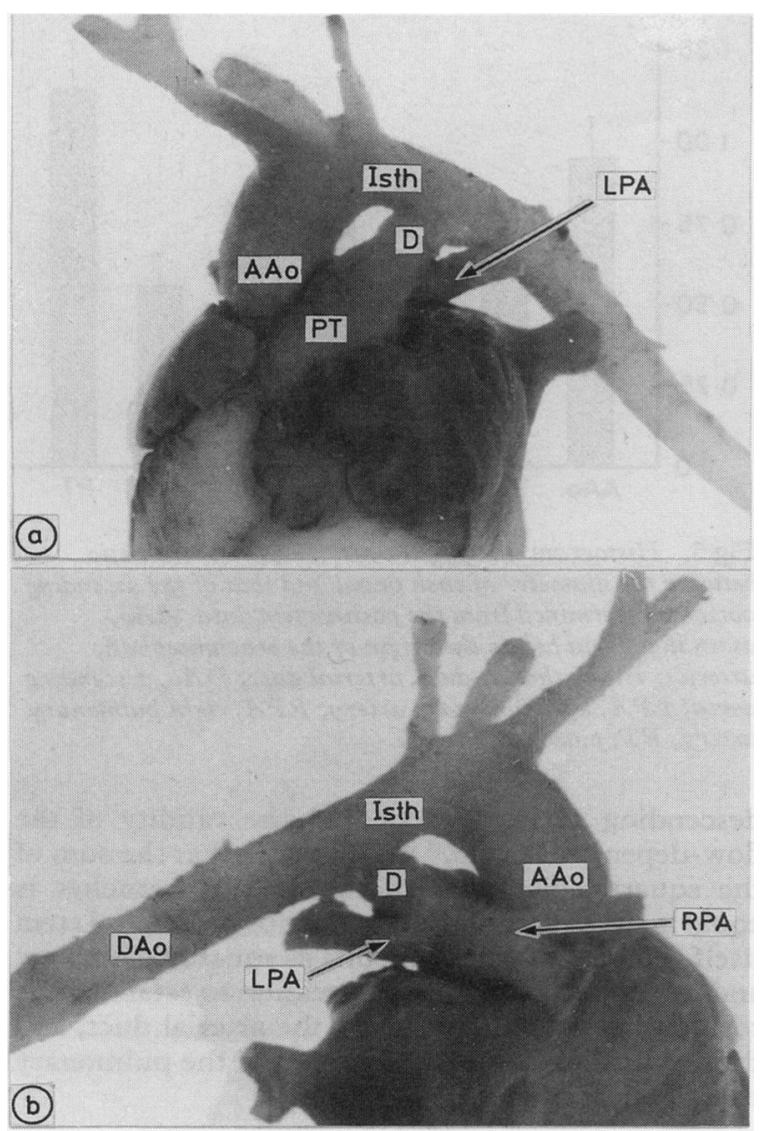

Fig 6 Heart and arch structures of a fetus of 20 weeks' gestation showing the size of the vessels. Note the absence of narrowing at the site of the isthmus. (a) Left anterior view and (b) right posterior view. See fig 5 for abbreviations.

of gestational age. The diameters of the ascending aorta and pulmonary trunk were measured at the level of the arterial valves, keeping the ultrasound beam perpendicular to the vessel walls wherever possible. The isthmus was measured as the smallest aortic diameter occurring immediately after the origin of the subclavian artery (fig 2). The right pulmonary artery and duct were measured at their origins (fig 3). Wherever possible we measured the duct and isthmus in the same projection and on the same stop frame (fig 4). We measured each vessel several times. Initially, five separate frames were measured and the results were averaged; these showed little deviation. Subsequently, therefore, three measurements of separate frames were taken at each level. The left pulmonary artery was not consistently imaged and, hence, was not included in the echocardiographic measurements. All measurements were taken by the same observer (LDA).

\section{Results}

POSTMORTEM DATA

In three specimens there was no discernible increas or decrease in the measured diameters of the vessels. before and after fixation. The mean ratio between the isthmus and the ascending aorta was $0.63(0.11)$ whild that between the arterial duct and the ascending aorty was $0.51(0.13)$ (fig $5 ; \mathrm{p}>0.001$ by Students $t$ test) ${ }_{0}$ The ratios between the left and right pulmonary arteries and the ascending aorta were $0.48(0.10)$ and $0.56(0.10)$ respectively (fig 5$)$. The isthmus was larger than the duct in 20 of the 30 cases (fig 6). . It was. smaller in seven of the 30 and the same in thre specimens. The right pulmonary artery was larget than the duct in 16, equal to the duct in six, and smaller in eight of the 30 . The left pulmonary artery was always smaller than the right pulmonary arteris but was larger than the arterial duct in eight of the $3 \theta$ specimens. The pulmonary trunk was larger than the ascending aorta in 24, of equal size in five, and smalle? in only one of the 30 specimens. The ascending aorta was larger than the descending aorta in 24 , equal in four, and smaller in two. Figure 7 shows the percen: tage difference between the diameter of each vessedo and that of the descending aorta. The diameters $0 \Phi^{\circ}$ the pulmonary trunk and the ascending aorta were $46.07 \%$ and $24.77 \%$ more than that of the descend

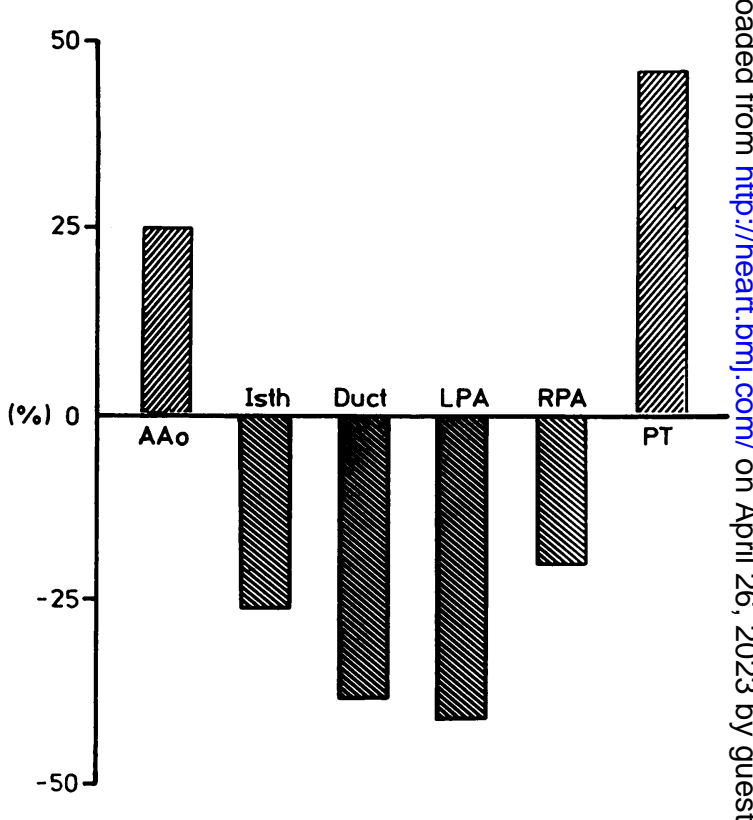

Fig 7 Histogram showing the percentage difference between the diameters of each vessel and that of the descending aorta as assessed from the postmortem data. See fig 5 for abbreviations. 


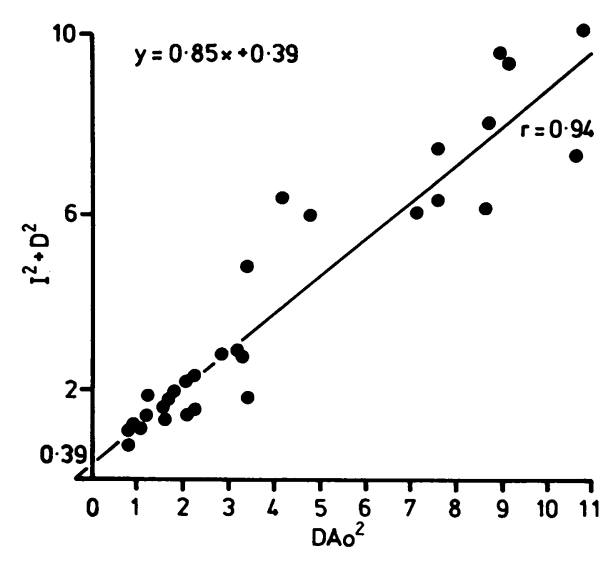

Fig 8 Regression line for the diameters of the descending aorta versus those of the arterial duct and isthmus expressed as squared diameters.

ing aorta. The diameter of the duct was $38 \cdot 26 \%$ smaller than the descending aorta and the isthmus was $24.57 \%$ smaller. Figures 8 and 9 show the calculations for verification of the flow-dependence rule. The regression coefficients for the relations between the descending aorta versus its branches $(r=0.94)$ and the pulmonary trunk versus its branches $(r=0.89)$ both showed a good correlation.

\section{ECHOCARDIOGRAPHIC DATA}

The ratio between the isthmus and the ascending aorta was $0.73(0.07)$ while that between the arterial duct and the ascending aorta was $0.7(0.08)$. These differences are not statistically significant. The ratio between the right pulmonary artery and the ascending aorta $(0.62(0.06))$ was similar to that of the duct (fig 10). The isthmus was found to be of larger diameter than the duct in 15 cases, of equal size in three, and smaller in only two cases. The right pulmonary artery was slightly smaller than the duct

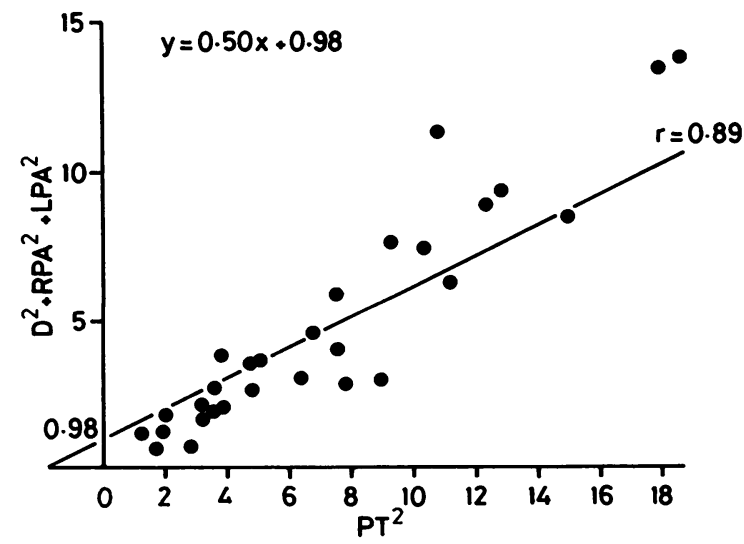

Fig 9 Regression line for the diameters of the pulmonary trunk versus those of the duct, and left and right pulmonary arteries expressed as squared diameters. in all but one case. The pulmonary trunk was larger than the aorta in all cases.

\section{Discussion}

The first morphometric data on the size of the human isthmus and arterial duct were based on angiographic and postmortem data. ${ }^{12}$ These studies were obtained in postnatal, premature, and full term neonates. These are the only detailed morphometric studies of the pathways of the human fetal circulation. Current concepts of human fetal circulation are derived from the work of Rudolph et al who measured flow in the fetal lamb. Despite acknowledging some drawbacks, this group postulated that these findings are applicable to the human fetus. ${ }^{10}$ Accordingly, it is held that the development of vessels is proportional to the amount of blood carried by them. Similar flow, therefore, should produce vessels of similar diameter. Yet recent reviews by Rudolph et al ${ }^{1012}$ conclude that the ascending aorta, which carries $35-40 \%$ of combined ventricular output, is similar in diameter to the descending aorta, which is presumed to carry about $70 \%$ of the combined ventricular output. The size of the ascending aorta is also thought to be comparable with that of the arterial duct (which carries $55 \%$ of the combined ventricular output) while the isthmus (10-15\% of the combined ventricular output) is said to have a cross sectional area that is half that of the descending aorta.

Recent developments in fetal echocardiography have now given us the opportunity of studying directly the dimensions of these vessels in the developing human fetus. Such measurements taken during fetal life are needed because fixation and agonal changes may have different effects on the calibre of elastic and muscular vessels ${ }^{13}$ and isolation of the specimen might also cause premature contraction of the arterial duct. The echocardio-

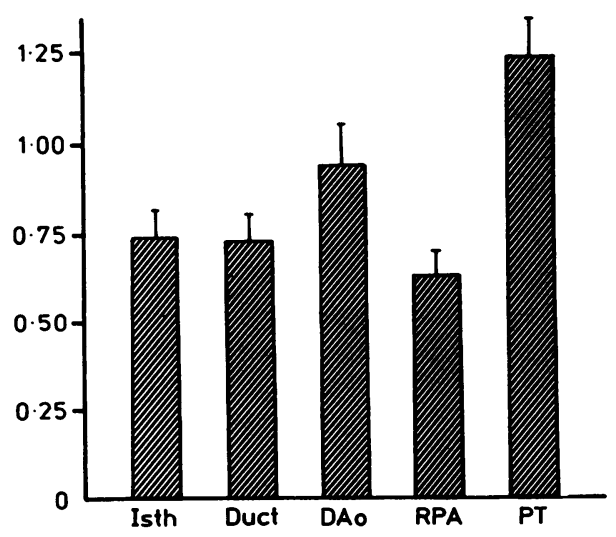

Fig 10 Histogram showing the mean (SD) of the ratio between the diameters of each vessel and that of the ascending aorta as calculated from the echocardiographic data. See fig 5 for abbreviations. 
graphic technique too has drawbacks. To minimise error, we chose patients with the best quality images and where possible made measurements in the axial plane because it gives better resolution than the lateral plane. In particular, we took care to make measurements of the isthmus and arterial duct in the same projection. Taken overall, none the less, all these limitations can be overcome if the echocardiographic and postmortem measurements are compared. We found that although the absolute results of these studies differed, the ratios of vessel size obtained with the two techniques are similar. Taken together, they show that the isthmus and the arterial duct are usually similar in size and that occasionally the duct is smaller. The calibres of the isthmus and arterial duct measured echocardiographically were not significantly different. In contrast, morphological measurements showed that the isthmus was significantly larger than the arterial duct $(p<0.001)$. The discrepancy might be the result of greater shrinkage of the muscular duct relative to the elastic isthmus during fixation. But measurements in fresh specimens show no change in trend when compared with the same specimens after fixation. Similar findings have previously been reported. ${ }^{14}$

Our results, therefore, show that the isthmus is not as small during the middle period of fetal life as was generally assumed from data derived from animal models. Our observations support the "flowdependence rule"11 - that the dimensions of main channels are proportional to the sums of the dimensions of their tributaries. We can make several propositions about the human fetus from our results. Our measurements show that the arterial duct has a smaller diameter at mid-gestation than previously thought. This suggests that flow through the human arterial duct may be less than previously assumed, or that the influence of flow on the size of the vessel is less. Alternatively, if the cardiac output from the right ventricle is as expected from animal models, then a greater amount of flow may go through the lungs. A further possibility is that the cardiac output from the right ventricle may be less, with flow to the pulmonary arteries being as previously thought.

Whatever the reasons, the aortic isthmus in the human fetus is larger and the arterial duct smaller than previously assumed. This suggests that the distribution of the circulation and the fetal flow patterns may be significantly different in the human fetus and the lamb fetus. In addition, it may be that the proportion of the cardiac output carried through the vessel may have a somewhat lesser effect on its size than previously thought. None the less, our study was performed on fetuses that were generally between the tenth and thirtieth weeks of gestation. Just as we have shown the dangers of extrapolating from animal models to the human, we caution against presuming that our conclusions can be applied to the later period of intrauterine life. Further studies are in progress to chart the dimensions of the arch vessels. during this crucial period.

We thank Dr Audrey Smith from the Royal Chil dren's Hospital, Liverpool and Dr R J Hangartners and all the medical staff at the Histopathology Department of St George's Hospital Medical School London for the supply of the specimens. We also thank Dr Cecille O Sunderland of the University of Oregon, Portland, for her critical review of an earlier $\vec{\omega}$ version of this paper.

AA was supported by a grant from the Ministry of Education, Italy, during the course of this investigation.

\section{References}

1 McNamara DG. Coarctation-course and prognosis in $\vec{p}$ infancy and childhood. In: Kidd BSL, Keith JD, eds 8 The natural history and progress in treatment of congenital heart defect. Springfield, Illinois: Charleș C Thomas, 1971:267-74.

2 Sinha SN, Kardatzke ML, Cole RB, Muster $\mathrm{AJ}_{2}^{\frac{\mathbb{N}}{-}}$ Wessel HU, Paul MH. Coarctation of the aorta in infancy. Circulation 1969;50:385-98.

3 Rudolph AM, Heymann MA, Teramo KAW, Barret CT, Raiha NCR. Studies on the circulation of the previable human fetus. Pediatr Res 1971;5:452-65.

4 Dawes GS, Mott JC, Widdicombe JG. The foeta circulation in the lamb. J Physiol (Lond) 1954年 126:563-87.

5 Assali NS, Kirschbaum TM, Dilts PV Jr. Effects of hyperbaric oxygen on uteroplacental and feta circulation. Circ Res 1968;22:573-88.

6 Peltonen T, Hirvonen L. Experimental studies on fetat and neonatal circulation. Acta Paediatr Scand 1965;suppl 161.

7 Heymann MA, Rudolph AM. Effect of exteriorization of the sheep fetus on its cardiovascular function. Ciré Res 1967;21:741-5.

8 Rudolph AM, Heymann MA. The circulation of the fetus in utero. Circ Res 1967;21:163-84.

9 Rudolph AM, Heymann MA. Circulatory changes during growth in the fetal lamb. Circ ReD

1970;26:289-99.
10 Rudolph AM, Heymann MA, Spitznas U을. Hemodynamic considerations in the development of narrowing of the aorta. Am J Cardiol 1972;30:514-25\%

11 Krediet $P$, Klein HW. Synopsis of normal cardiad development. In: Pexieder T, ed. Perspectives inN cardiovascular research. New York: Raven Press

12 Rudolph AM. Distribution and regulation of blood flok in the fetal and neonatal lamb. Circulation 1985 57:811-21.

13 Hornblad PY, Larsson KS. Studies on closure of the् ductus arteriosus. Cardiologia 1967;51:231-41.

14 Van Meurs-Van Woezik H, Klein HW. Calibres of aorta and pulmonary artery in hypoplastic left an $\Phi^{\circ}$ right heart syndromes: effects of abnormal bloodflow? Virchows Arch [A] 1974;364:357-64. 